\title{
First-Principles Study on Electronic and Magnetic Properties of Sc-, Ti- and Zn-Doped Graphene
}

\author{
A.E. Auguscik, A.P. Durajski* and R. Szcześsiniak \\ Institute of Physics, Częstochowa University of Technology, al. Armii Krajowej 19, 42-200 Częstochowa, Poland
}

\begin{abstract}
Graphene is an attractive candidate for the future generation of nanoelectronic and nanophotonic devices, gas sensors, biosensors, and batteries for energy storage. The fact that pristine graphene is not magnetic limits its application in areas such as magnetic storage and spintronics. However, recent theoretical and experimental results indicate that adsorption of metal adatoms and substitutional doping are promising ways to modulate the electronic properties and induce a magnetic state of graphene-based systems. Using the density functional theory we study the properties of graphene sheets substitutionally doped with $\mathrm{Sc}$, $\mathrm{Ti}$, and $\mathrm{Zn}$ atoms. In particular, the influence of metal doping on geometric structure, electronic structure, and magnetic behavior of graphene were analysed. The obtained results indicate that the optimized geometries show distortion on doping and the electronic structures are significantly modified. To examine the magnetic state of doped graphene both ferromagnetic and antiferromagnetic cases were taken into consideration.
\end{abstract}

DOI: 10.12693/APhysPolA.137.958

PACS/topics: graphene, doping, electronic properties, magnetic properties, DFT calculations

\section{Introduction}

Graphene is a sheet of bonded carbon atoms arranged in a honeycomb structure. This two-dimensional material was discovered in 2004 by Geim and Novoselov as a result of micromechanical cleavage of carbon atoms layers from graphite [1]. Due to the exceptional properties of graphene this material has attracted increasing attention and is believed to be a promising candidate for the future generation of nanoelectronic and nanophotonic devices, gas sensors, biosensors, and batteries for energy storage [2]. However, the lack of band gap has greatly limited the potential application of graphene. One of the ways to circumvent this issue is opening a band gap in graphene through its physical and chemical modifications $[3,4]$. The interatomic bonds with $s p^{2}$ hybridization enable alteration of graphene systems and connection with other chemical elements, which change its properties. Graphene intercalation compounds show various fascinating physical properties that are lacking in pristine graphene. The recent studies of graphene doping with the alkali metals have brought up new exciting results, like superconductivity in low-dimensional systems [5-8]. Moreover, the theoretical and experimental results indicate that adsorption of metal atoms and substitution doping is a promising way to tunable electronic properties and induce the magnetic state in graphene-based systems [9-11].

In this paper, first-principles calculations were adopted to evaluate the properties of graphene doped with $3 d$ metal atoms (scandium, titanium, and zinc). In particular, the influence of metal doping on the geometrical

*corresponding author; e-mail: adurajski@wip.pcz.pl structure, electron structure, and magnetic behavior of graphene were analyzed. The obtained results indicate that optimized geometries exhibit distortion doping and electronic structures are significantly modified. Ferromagnetic and antiferromagnetic states were investigated, which allowed concluding that the Zn-doped graphene supercell exhibits antiferromagnetic ordering.

\section{Computational methods}

In order to characterize the metal-doped graphene, we performed first-principles density functional theory (DFT) simulations within the generalized gradient approximation of the Perdew-Burke-Ernzerhof (GGA-PBE) approach as implemented in the Quantum Espresso package [12]. The ground state of investigated system is obtained from the solutions of the single particle Kohn-Sham equations

$$
\left(-\frac{\hbar^{2}}{2 m} \nabla^{2}+V_{\mathrm{ext}}(\boldsymbol{r})+V^{(\mathrm{in})}(\boldsymbol{r})\right) \psi_{i}(\boldsymbol{r})=\epsilon_{i} \psi_{i}(\boldsymbol{r}),
$$

where $\epsilon_{i}$ and $\psi_{i}$ are the Kohn-Sham energies and orbitals respectively, $i$ labels the occupied states, $V_{\text {ext }}$ is the external potential, the input Hartree and exchange-correlation potential $V^{(\text {in) }}(\boldsymbol{r})=V_{H x c}\left[\rho^{(\mathrm{in})}(\boldsymbol{r})\right]$ is a functional of the input charge density $\rho^{(\text {in) }}$. The kinetic energy cutoff for the wave function is set to 40 Ry and the kinetic energy cutoff for charge density is $400 \mathrm{Ry}$. The investigated systems consist of a $6 \times 6$ supercell of graphene (containing $72 \mathrm{C}$ atoms) with four $\mathrm{C}$ atoms substituted by four $3 d$ metal atoms (5.5\% dopant concentration). To avoid the interaction between single graphene planes a vacuum of $12 \AA$ between the nearest-neighbours layers was assumed. All the structures were fully relaxed until the forces was less than $0.01 \mathrm{eV} / \AA$. The Brillouin zone is sampled utilizing a $8 \times 8 \times 1 \boldsymbol{k}$-mesh in the MonkhorstPack scheme and the Marzari-Vanderbilt cold smearing 
with a smearing factor of $0.005 \mathrm{Ry}$ is used in all calculations. A $32 \times 32 \times 1$ Monkhorst-Pack grid is applied for the calculation of density of states (DOS).

\section{Results and discussion}

The doped graphene structures were created by using $6 \times 6$ supercell of pristine graphene and by removing four symmetrically situated $\mathrm{C}$ atoms. Thereafter at these vacant sites, foreign atoms $(\mathrm{Sc}, \mathrm{Ti}$, or $\mathrm{Zn})$ were embedded and the obtained structures were relaxed to find the most energetically favorable atomic positions and possible bonding (see Fig. 1). The $\mathrm{C}-\mathrm{C}$ nearest-neighbor bond length in pristine graphene is $1.42 \AA$. When four $\mathrm{Sc}$, Ti or $\mathrm{Zn}$ atoms replace four $\mathrm{C}$ atoms, as shown in Fig. 1, the geometric structure of initially graphene monolayer undergoes a significant rearrangement, due to the much larger size of dopant atoms with respect to carbon.

The metal atoms protrude out of the graphene plane at a distance $h$ of $1.67,1.36$, and $1.38 \AA$ and the three nearest-neighbor $\mathrm{C}$ atoms are lifted up from the plane by $0.29,0.21$, and $0.30 \AA$ for $\mathrm{Sc}, \mathrm{Ti}$, and $\mathrm{Zn}$ impurities, respectively. The $\mathrm{Sc}-\mathrm{C}, \mathrm{Ti}-\mathrm{C}$ and $\mathrm{Zn}-\mathrm{C}$ bond lengths are $2.06,1.92$, and $1.93 \AA$ and the $\mathrm{C}-\mathrm{Sc}-\mathrm{C}, \mathrm{C}-\mathrm{Ti}-\mathrm{C}$, and $\mathrm{C}-\mathrm{Zn}-\mathrm{C}$ bond angles are $80.01^{\circ}, 87.35^{\circ}$, and $91.94^{\circ}$, respectively.

The binding energy $\left(E_{b}\right)$ can then be estimated by

$$
E_{b}=E_{\text {graphene }}+E_{\text {metal }}-E_{\text {graphene+metal }},
$$

where $E_{\text {graphene }}$ is the total energy of the graphene with a vacancy, $E_{\text {metal }}$ is the ground state energy of an isolated dopant atom, and $E_{\text {graphene+metal }}$ represents the total energy of the doped graphene system. All energies were calculated for the fixed supercells. The obtained binding energy for Sc-, Ti-, and $\mathrm{Zn}$-doped graphene is $6.40,7.97$, and $1.30 \mathrm{eV}$, respectively, which is in good agreement with the previous results reported by Krasheninnikov et al. [13]. Hence, we can deduce that a strong covalent interaction between the $\mathrm{Sc} / \mathrm{Ti}$ atoms and its nearest-neighbor $\mathrm{C}$ atoms indicates that Sc- and Tidoped graphene systems are more stable than Zn-doped case. Moreover, to have a view of the charge transfer of doped metal atoms, the charge difference was calculated using the expression

$$
\Delta \rho=\rho_{\text {graphene }+ \text { metal }}-\rho_{\text {metal }}-\rho_{\text {graphene }},
$$

where $\rho_{\text {graphene+metal }}, \rho_{\text {metal }}$, and $\rho_{\text {graphene }}$ are the charge densities of metal-doped graphene system, isolated metal atoms, and the graphene monolayer with vacancies, respectively. The charge difference plots of $\mathrm{Sc}-, \mathrm{Ti}-$, and Zn-doped graphene are shown in Fig. 2, with blue and yellow areas representing the electron loss and gain, respectively. As the electronegativities of $\mathrm{Sc}, \mathrm{Ti}$, and $\mathrm{Zn}$ are lower than that of $\mathrm{C}$, an obvious net gain of charges around $\mathrm{Sc}, \mathrm{Ti}$, and $\mathrm{Zn}$ atoms and a net loss of charges above graphene monolayer are found, indicating a significant charge transfer from the substrate to the metal atoms.
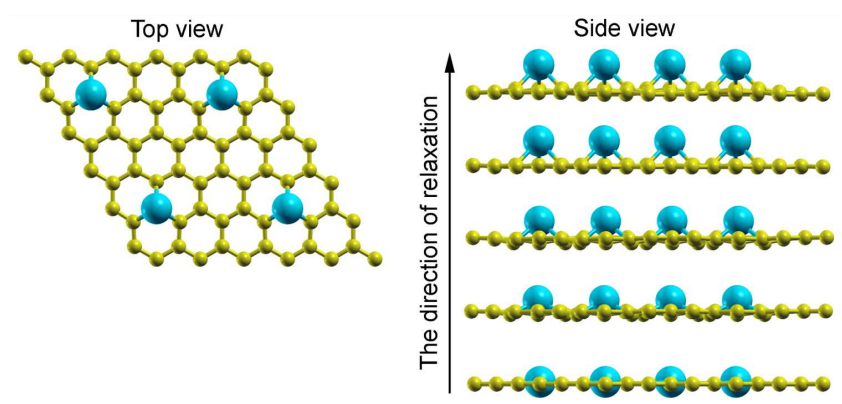

Fig. 1. Top view of the Sc-doped graphene structure and the side view of the evolution of the geometrics of Sc-doped graphene during the relaxation procedure. The $\mathrm{C}$ atoms are shown in yellow and the Sc atoms are shown in cyan.

(a)
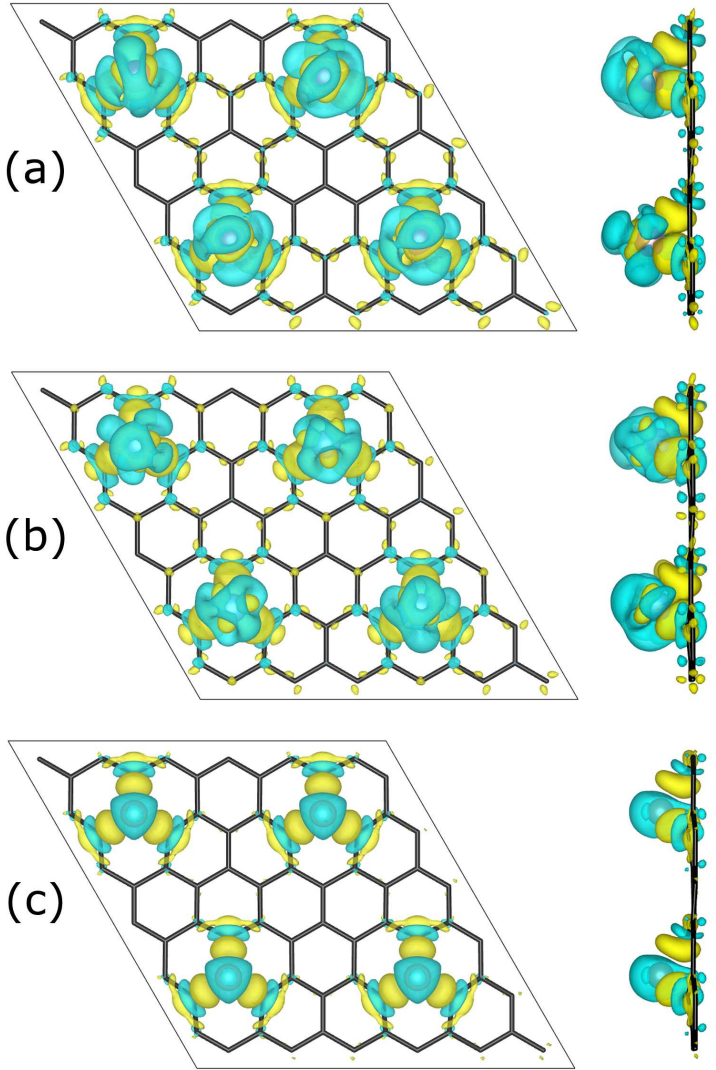

Fig. 2. The top and side view of charge density difference diagrams for (a) Sc-, (b) Ti- and (c) Zn-doped graphene with a charge density iso-surface value of $0.004 e / a_{0}{ }^{3}$, where $a_{0}$ is the Bohr radius. The blue and yellow areas represent the electron loss and gain.

The density of states (DOS) has been calculated to investigate the electronic properties of graphene with transition metal atoms as substitutional impurities. Figure 3 demonstrates the results for the pristine graphene supercell (dashed lines) and, subsequently, results obtained for doped systems. As we can see, after doping the electronic 

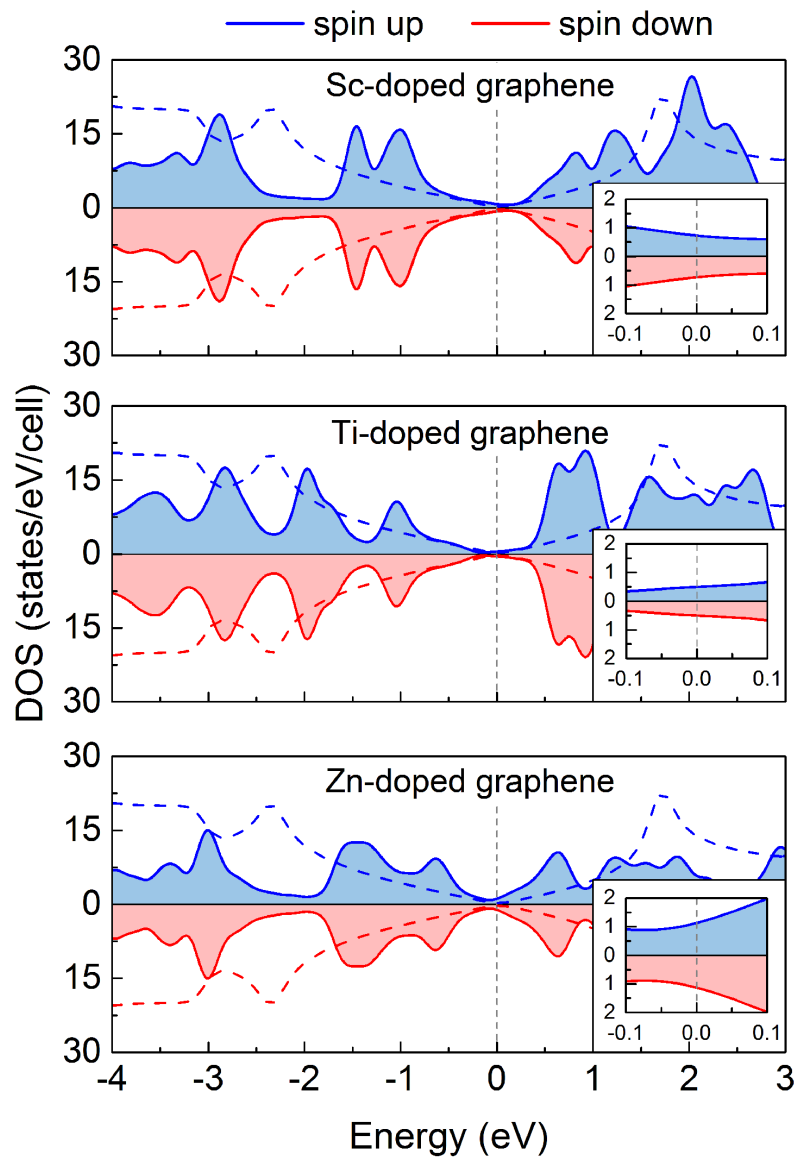

Fig. 3. A comparison of the spin-polarized density of states (DOS) of pristine graphene (dashed lines) and Zn-, Ti- and Sc-doped graphene supercells.

structure of graphene was significantly changed. To examine the magnetic behavior of doped graphene we calculated the energy difference between the ferromagnetic $(\mathrm{FM})$ and antiferromagnetic (AFM) state. We find that only the $6 \times 6$ graphene supercell with Zn-doping has evident magnetism (antiferromagnetic state). In this case, the AFM state is more stable than FM because their energy at AFM state $\left(E_{\mathrm{AFM}}\right)$ is lower than that at FM state $\left(E_{\mathrm{FM}}\right)$, in particular: $E_{\mathrm{FM}}-E_{\mathrm{AFM}}=-0.0035 \mathrm{Ry}$. In other cases, the difference is smaller than $10^{-9} \mathrm{Ry}$.

\section{Conclusions}

Using the first-principles methods, we studied the geometric structure, electronic properties, and magnetic behavior of Sc-, Ti-, and Zn-doped graphene supercells. The obtained results indicate that the optimized geometries show distortion on doping and the electronic structures are significantly modified. From our analysis, the interactions between zinc atoms and carbon atoms lead to the antiferromagnetism.

\section{Acknowledgments}

A.P. Durajski acknowledges the financial support of the Częstochowa University of Technology under Grant No. BS/MN-200-301/2019 and from the Polish Ministry of Science and Higher Education under the scholarship for young outstanding scientists No. 406/STYP/13/2018. This research was supported in part by PLGrid Infrastructure.

\section{References}

[1] K.S. Novoselov, A.K. Geim, S.V. Morozov, D. Jiang, Y. Zhang, S.V. Dubonos, I.V. Grigorieva, A.A. Firsov, Science 306, 666 (2004).

[2] H.R. Jiang, W. Shyy, M. Liu, L. Wei, M.C. Wu, T.S. Zhao, J. Mater. Chem. A 5, 672 (2017).

[3] G. Giovannetti, P.A. Khomyakov, G. Brocks, P.J. Kelly, J. van den Brink, Phys. Rev. B 76, 073103 (2007).

[4] J. Pesic, V. Damljanovic, R. Gajic, K. Hingerl, M. Belic, Europhys. Lett. 112, 67006 (2015).

[5] J. Pesic, R. Gajic, K. Hingerl, M. Belic, Europhys. Lett. 108, 67005 (2014).

[6] D.M. Guzman, H.M. Alyahyaei, R.A. Jishi, 2D Materials 1, 021005 (2014).

[7] A.P. Durajski, K.M. Skoczylas, R. Szczesniak, Phys. Chem. Chem. Phys. 21, 5925 (2019).

[8] A.P. Durajski, K.M. Skoczylas, R. Szczęśniak, Supercond. Sci. Technol. 32, 125005 (2019).

[9] J. Dai, J. Yuan, J. Phys. Condens. Matter 22, 225501 (2010).

[10] M. Sun, W. Tang, Q. Ren, Y. Zhao, S. Wang, J. Yu, Y. Du, Y. Hao, Physica E 80, 142 (2016).

[11] S. Haffad, L. Benchallal, L. Lamiri, F. Boubenider, H. Zitoune, B. Kahouadji, M. Samah, Acta Phys. Pol. A 133, 1307 (2018).

[12] P. Giannozzi, O. Andreussi, T. Brumme, et al., J. Phys. Condens. Matter 29, 465901 (2017).

[13] A.V. Krasheninnikov, P.O. Lehtinen, A.S. Foster, P. Pyykkö, R.M. Nieminen, Phys. Rev. Lett. 102, 126807 (2009). 